\title{
Increased Piezo1 channel activity in interstitial Cajal-like cells induces bladder hyperactivity by functionally interacting with NCX1 in rats with cyclophosphamide- induced cystitis
}

Qian Liu', Bishao Sun¹, Jiang Zhao', Qingqing Wang ${ }^{1}$, Fan $\mathrm{An}^{2}$, Xiaoyan $\mathrm{Hu}^{1}$, Zhenxing Yang ${ }^{1}$, Jie Xu', Mingjia $\operatorname{Tan}^{3}$ and Longkun $\mathrm{Li}^{1}$

\begin{abstract}
The Piezo1 channel is a mechanotransduction mediator, and Piezo1 abnormalities have been linked to several clinical disorders. However, the role of the Piezo1 channel in cystitis-associated bladder dysfunction has not been documented. The current study aimed to discover the functional role of this channel in regulating bladder activity during cyclophosphamide (CYP)-induced cystitis. One hundred four female rats were randomly assigned to the control, CYP-4h, CYP-48h and CYP-8d groups. CYP successfully induced acute or chronic cystitis in these rats. CYP treatment for $48 \mathrm{~h}$ or $8 \mathrm{~d}$ significantly increased Piezo1 channel expression in bladder interstitial Cajal-like cells (ICC-LCS), and the increase in CYP-8d rats was more prominent. In addition, $2.5 \mu \mathrm{M}$ Grammostola spatulata mechanotoxin 4 (GsMTX4) significantly attenuated bladder hyperactivity in CYP-8d rats by inhibiting the Piezo1 channel in bladder ICCLCs. Furthermore, by using GsMTx4 and siRNA targeting the Piezo1 channel, we demonstrated that hypotonic stressinduced Piezo1 channel activation significantly triggered $\mathrm{Ca}^{2+}$ and $\mathrm{Na}^{+}$influx into bladder ICC-LCs during CYPinduced chronic cystitis. In addition, the Piezo1 channel functionally interacted with the relatively activated reverse mode of $\mathrm{Na}^{+} / \mathrm{Ca}^{2+}$ exchanger 1 (NCX1) in bladder ICC-LCS from CYP-8d rats. In conclusion, we suggest that the functional role of the Piezo1 channel in CYP-induced chronic cystitis is based on its synergistic effects with NCX1, which can significantly enhance $\left[\mathrm{Ca}^{2+}\right]_{i}$ and result in $\mathrm{Ca}^{2+}$ overload in bladder ICC-LCS, indicating that the Piezo1 channel and NCX1 are potential novel therapeutic targets for chronic cystitis-associated bladder hyperactivity.
\end{abstract}

\section{Introduction}

Interstitial cystitis/painful bladder syndrome (IC/PBS) is a clinical syndrome characterized by chronic pelvic pain

\footnotetext{
Correspondence: Mingjia Tan (mjtan@umich.edu) or Longkun Li

(lilongk@hotmail.com)

'Department of Urology, Second Affiliated Hospital, Third Military Medical

University, Chongqing 400037, China

${ }^{2}$ Cancer Center, Institute of Cancer Stem Cell, Dalian Medical University, Dalian

116044, China

Full list of author information is available at the end of the article
}

and other micturition symptoms, such as frequency, urgency, and nocturia ${ }^{1}$. The prevalence of IC/PBS ranges from 1 in 100000 to 5.1 in 1000 in the worldwide population $^{2}$. The etiology and pathophysiology of IC/BPS are poorly understood, and the therapeutic strategies against this disorder are still limited and unsatisfactory ${ }^{3}$. Therefore, more research is needed to clarify the pathogenesis of IC/BPS

In recent years, increasing evidence has demonstrated that bladder interstitial Cajal-like cells (ICC-LCs), which

\section{(c) The Author(s) 2018}

\footnotetext{
Open Access This article is licensed under a Creative Commons Attribution-NonCommercial-NoDerivatives 4.0 International License, which permits any non-commercial CC. Creative Commons license. You do not have permission under this license to share adapted material derived from this article or parts of it. The images or other third party material in this article are included in the article's Creative Commons license, unless indicated otherwise in a credit line to the material. If material is not included in the article's Creative Commons license and your intended use is not permitted by statutory regulation or exceeds the permitted use, you will need to obtain permission directly from the copyright holder. To view a copy of this license, http:// creativecommons.org/licenses/by-nc-nd/4.0/.
} 
display many of the morphological features of gastrointestinal interstitial Cajal cells (ICCs), play a crucial role in regulating bladder activity ${ }^{4-8}$. Two populations of ICCLCs have been identified, including ICC-LCs in the lamina propria (LP, area between the urothelium and detrusor smooth muscle) and ICC-LCs in the detrusor (intramuscular and interbundle) ${ }^{9}$. The functional character of bladder ICC-LCs has not been completely clarified. The common hypothesis is that ICC-LCs in the LP primarily play a role in nonneural sensory signal transduction from the urothelium to the detrusor, whereas detrusor ICC-LCs might be involved in pacemaking and/ or transduction of pacemaking signals in the detrusor ${ }^{10}$. It has been shown that abnormalities in bladder ICC-LC quantity and function are involved in several bladder disorders, such as bladder outlet obstruction, diabetic cystopathy, and neurogenic bladder ${ }^{11}$. The data on functional changes in IC/PBS are still limited. Although we have found that bladder ICC-LC hyperactivity induced by alterations in the hyperpolarization-activated cyclic nucleotide 1 (HCN1) channel are involved in cyclophosphamide (CYP)-induced cystitis $^{12}$, investigations attempting to more comprehensively elucidate the mechanisms underlying the functional role of bladder ICC-LCs in cystitis will be beneficial in developing more therapeutic strategies for cystitis-associated bladder dysfunction.

Piezo proteins, including Piezo1 and Piezo2, have recently been identified as mechanically activated cation channels in mammalian cells ${ }^{13}$. In vertebrates, the Piezo2 channel mediates gentle touch sensation, whereas the Piezo1 channel has multiple roles in various physiological processes, including regulating vascular development, controlling cell migration and differentiation, maintaining blood pressure, and triggering rapid epithelial cell divi$\operatorname{sion}^{14-16}$. Aberrant Piezo1 channel activity has been linked to several diseases, such as hereditary xerocytosis ${ }^{17}$, ${ }^{18}$, generalized lymphatic dysplasia ${ }^{19}$, and heart failure ${ }^{20}$. In the bladder, the Piezol channel is found to be associated with the $\mathrm{Ca}^{2+}$ influx and ATP release in urothelial cells $^{21}$, and its expression is significantly increased in rats after partial bladder outlet obstruction $(\mathrm{PBOO})^{22}$. The functional changes in the Piezo1 channel in other bladder dysfunctions, such as IC, need to be further explored.

To date, several animal models that mimic IC/BPS symptoms have been well-established using CYP, potassium chloride $(\mathrm{KCl})$, protamine sulfate, lipopolysaccharide (LPS), and immunogenic peptide, among others ${ }^{23-26}$. Among these, CYP-induced cystitis is one of the most commonly used models ${ }^{23}$, 27. In this study, we used this model and demonstrated that increased Piezo1 channel activity in bladder ICC-LCs is a crucial factor for CYPinduced cystitis-associated bladder hyperactivity. The functional role of the Piezo1 channel is based on its synergistic effects with $\mathrm{Na}^{+} / \mathrm{Ca}^{2+}$ exchanger 1 (NCX1), which significantly enhances the intracellular $\mathrm{Ca}^{2+}$ concentration, leading to $\mathrm{Ca}^{2+}$ overload in bladder ICC-LCs.

\section{Materials and methods \\ Animals}

Female Sprague-Dawley (SD) rats (220-250 g) were purchased from Experimental Animals Center of Third Military Medical University (Chongqing, China). Initially, 24 rats were randomly assigned to control, CYP-4h, CYP$48 \mathrm{~h}$, and CYP-8d groups. For further research, 80 rats were randomly assigned into the control and CYP-8d groups. All rats were maintained in light-controlled (12-h light/dark cycle) cages with free access to rodent chow and water. All experimental procedures were approved by the Research Council and Animal Care and Use Committee of the Third Military Medical University, China and were performed in accordance with the Guide for the Care and Use of Laboratory Animals published by the National Institutes of Health.

\section{Induction of CYP-induced cystitis}

Rat models of CYP-induced cystitis were established according to previously described protocols ${ }^{28,29}$. To induce acute cystitis, rats in the CYP-4h and CYP-48h groups received a single administration $(150 \mathrm{mg} / \mathrm{kg}$; intraperitoneally) of CYP (Sigma, St Louis, MO, USA) and were sacrificed 4 or $48 \mathrm{~h}$ after CYP administration. Chronic cystitis was induced by three injections of lowdose CYP. Rats in the CYP-8d and control groups received the administration of CYP $(75 \mathrm{mg} / \mathrm{kg}$; intraperitoneally) or an equal volume of vehicle (sterile saline) on days 1,4 , and 7 and were sacrificed $24 \mathrm{~h}$ after the last CYP administration.

\section{Hematoxylin and eosin (H\&E) staining}

Bladders isolated from sacrificed rats were sequentially fixed with $4 \%$ paraformaldehyde, dehydrated, embedded in paraffin, and cut into $4-\mu \mathrm{m}$ sections. Then, the sections were deparaffinized and rehydrated in a graded ethanol series. $H \& E$ staining was performed according to a standard protocol ${ }^{30}$.

\section{Western blot analysis}

Rat bladders were lysed in RIPA lysis buffer (Beyotime, Shanghai, China) to extract total protein. Protein concentrations were measured with a Bio-Rad DC Protein Assay Kit (Bio-Rad, Hercules, USA). Then, $50 \mu$ g protein aliquots were separated using $8-12 \%$ sodium dodecyl sulfate polyacrylamide gel electrophoresis (SDS-PAGE) gels and transferred to polyvinylidene fluoride (PVDF) membranes (Merck Millipore, Darmstadt, Germany). After being blocked with 5\% skim milk dissolved in Trisbuffered saline at room temperature for $2 \mathrm{~h}$, the 
membranes were incubated overnight at $4{ }^{\circ} \mathrm{C}$ with various primary antibodies targeting the following proteins: IL-6 (Abcam, Cambridge, UK, ab9324, 1:800), TNF- $\alpha$ (Abcam, ab199013, 1:500), Piezo1 (Alomone labs, Jerusalem, Israel, APC-087, 1:500), NCX1 (Abcam, ab2869, 1:800), NCX2 (Alomone labs, ANX-012, 1:500), NCX3 (Alomone labs, ANX-013, 1:500), glyceraldehyde-3-phosphate dehydrogenase (GAPDH) (Beyotime, AG019, 1:1000), and $\alpha$ tubulin (Beyotime, AT819, 1:1000). Following incubation with horseradish peroxidase-conjugated secondary antibodies (Zhongshan Co., Beijing, China, ZB-2301, ZB2305, 1:5000), antibody-antigen complexes were detected using an ECL substrate (Millipore, Billerica, USA) and visualized with an Image Quant LAS-4000 BioImaging System (GE Healthcare, Stockholm, Sweden).

\section{Quantitative reverse transcription-PCR (RT-PCR)}

Total RNA was isolated from rat bladders using TRIzol reagent (Takara, Dalian, China) according to the manufacturer's protocol. Total RNA $(2 \mu \mathrm{g})$ was subsequently reverse transcribed to complementary DNA (cDNA) using a PrimeScript ${ }^{\mathrm{mi}}$ RT reagent kit (Takara). The primers used in our study are listed in Table S1. Quantitative real-time PCR was performed using SYBR Green mix (Toyobo, Osaka, Japan) in a StepOnePlus Real-Time PCR system (Life Technologies, Carlsbad, CA). The thermocycling program was run as follows: $95^{\circ} \mathrm{C}$ for $30 \mathrm{~s}, 95^{\circ} \mathrm{C}$ for $5 \mathrm{~s}, 60^{\circ} \mathrm{C}$ for $10 \mathrm{~s}$, and $72^{\circ} \mathrm{C}$ for $15 \mathrm{~s} \mathrm{(40} \mathrm{cycles).} \mathrm{Gene}$ expression was normalized to GAPDH expression and the fold changes were calculated using the $2-\triangle{ } \mathrm{Ct}$ method. The negative control was performed without templates.

\section{Immunohistochemistry (IHC)}

For IHC, bladder sections stained with H\&E were incubated overnight at $4{ }^{\circ} \mathrm{C}$ with primary antibody against Piezo1 (Alomone labs, APC-087, 1:100). Phosphatebuffered saline (PBS) was substituted for the primary antibody as a negative control. Following incubation with primary antibody, the sections were incubated with a streptavidin-biotin peroxidase (SP)-conjugated secondary goat anti-rabbit IgG from a standard SP kit (Zhongshan Co., SPN-9002) for $30 \mathrm{~min}$ at $37^{\circ} \mathrm{C}$. Finally, the sections were stained with diaminobenzidine (DAB) (Zhongshan Co., ZLI-9019) and counterstained with hematoxylin. All sections were visualized and photographed with an optical microscope (Olympus) and evaluated by two individuals in a double-blind manner.

\section{Immunofluorescence (IF) staining}

After being snap frozen, the isolated rat bladders were cut into $6-\mu \mathrm{m}$ sections on a freezing microtome. For IF staining, these tissue sections and cells on coverslips were fixed with $4 \%$ paraformaldehyde (Boster, Wuhan, China) for $30 \mathrm{~min}$ and then washed in PBS (10 $\mathrm{min} \times 3$ times).
After being blocked with immunostaining blocking buffer (Beyotime, Shanghai, China) for $1 \mathrm{~h}$, tissue sections and cells were incubated overnight at $4{ }^{\circ} \mathrm{C}$ with primary antibodies targeting the following proteins: C-kit (Santa Cruz, Dallas, TX, USA, sc-1494, 1:50), Piezo1 (Alomone labs, APC-087, 1:100) and NCX1 (Abcam, ab2869, 1:100), or with PBS as the negative control. Afterward, the tissue sections and cells were incubated at room temperature for $90 \mathrm{~min}$ with appropriate secondary antibodies (Alexa 488conjugated mouse anti-goat IgG (Bioss, Beijing, China, 1:200) or goat anti-mouse IgG (Beyotime, 1:200) and Alexa 647-conjugated donkey anti-mouse IgG or donkey anti-rabbit IgG (Bioss, 1:200)) and then incubated with 2(4-amidinophenyl)-6-indolecarbamidine dihydrochloride (DAPI, Beyotime) to label cytoblasts. Each incubation step was followed by PBS washes ( $5 \mathrm{~min} \times 3$ times). All tissue sections and cells were viewed and photographed with a laser-scanning confocal microscope (Leica, Wetzlar and Mannheim, Germany).

\section{Urodynamic measurements}

Urodynamic measurements were performed in unconscious rats as previously described ${ }^{31}$. Rats were anesthetized with an intraperitoneal injection of urethane $(1 \mathrm{~g} /$ $\mathrm{kg})$. A PE-50 polyethylene catheter was inserted into the bladder dome via a mid-line abdominal incision, and a purse-string suture was utilized to close the bladder dome incision tightly. The other end of the PE-50 catheter (Becton Dickinson \& Company, Franklin Lakes, New Jersey, USA) was connected to a pressure transducer (Chengyi Co., Chengdu, China) and an infusion pump (AVI 270, 3 M, Saint Paul, MN, USA) with a three-way connector. Physiological saline containing Grammostola spatulata mechanotoxin 4 (GsMTx4, Piezo1 antagonist, $2.5 \mu \mathrm{M}, \mathrm{Abcam}$ ) or dimethyl sulfoxide (DMSO) (Beyotime) at room temperature was infused into the bladder at a constant rate $(10 \mathrm{~mL} / \mathrm{h})$. Continuous urodynamic curves were digitized and recorded using a multichannel signal processing system (RM6240C, Chengyi Co.). The maximum bladder pressure (MBP) and intercontractile interval (ICI) were analyzed, which represent the bladder contractility and voiding frequency, respectively.

\section{Contractility studies}

The whole bladder was carefully resected from each sacrificed rat and placed in Kreb's solution containing the following compounds (in $\mathrm{mM}$ ): $119 \mathrm{NaCl}, 4.7 \mathrm{KCl}, 1.2$ $\mathrm{KH}_{2} \mathrm{PO}_{4}, 1.2 \mathrm{MgSO}_{4} \bullet 7 \mathrm{H}_{2} \mathrm{O}, 25 \mathrm{NaHCO}_{3}, 2.5 \mathrm{CaCl}_{2}$ and 11 glucose, adjusted to $\mathrm{pH} 7.4$ with $\mathrm{NaOH}$. Then, bladders were longitudinally cut into $3 \times 4 \times 8 \mathrm{~mm}$ strips. Each strip was suspended vertically in a $15-\mathrm{mL}$ organ bath filled with $37^{\circ} \mathrm{C}$ Kreb's solution and maintained in a mixture of $95 \% \mathrm{O}_{2}$ and $5 \% \mathrm{CO}_{2}$. One end of each strip was fixed to the bottom of the bath, and the other end was linked with the tension 
transducer. After equilibration for $30 \mathrm{~min}, 0.75 \mathrm{~g}$ of tension was applied to each strip to elicit spontaneous contraction, and stable continuous curves were visualized and recorded in the RM6240C signal processing system (Chengyi Co.). Pharmaceuticals, including GsMTx4 $(2.5 \mu \mathrm{M})$, the c-kit blocker imatinib mesylate (Glivec, $100 \mu \mathrm{M}$, Sigma, St Louis, MO, USA), the NCX reverse mode inhibitor (KBR7943, $10 \mu \mathrm{M}$, Sigma) and the vehicle (DMSO), were added to the bathing solution at 6-min intervals.

\section{Bladder ICC-LC isolation}

Rat bladders were aseptically resected and washed in sterile $\mathrm{Ca}^{2+}$-free Hank's solution (Boster). After the urothelium layer was removed, bladders were cut into small fragments and digested at $37^{\circ} \mathrm{C}$ for $5 \mathrm{~min}$ in $5 \mathrm{~mL}$ of enzyme solution containing the following reagents $(\mathrm{mg} /$ $\mathrm{mL}$ ): 2.0 type II collagenase, 2.0 bovine serum albumin (BSA) and 2.0 trypsin inhibitor (all were purchased from Sigma). To terminate digestion, RMPI-1640 medium (HyClone, Logan, USA) containing 10\% fetal bovine serum (Gibco-Life Technologies, Grand Island, NY, USA) was added to the enzyme solution. Afterward, the isolated cells were filtered via a 200-mesh cell strainer and plated onto sterile polylysine-coated glass coverslips or laser confocal dishes. The cells were cultured at $37^{\circ} \mathrm{C}$ in a $95 \%$ $\mathrm{O}_{2}$ and $5 \% \mathrm{CO}_{2}$ incubator in RMPI-1640 medium supplemented with $10 \%$ fetal bovine serum and $1 \%$ antibiotics/antimycotics (Beyotime).

\section{Measurement of intracellular calcium and sodium concentrations $\left(\left[\mathrm{Ca}^{+}\right]_{\mathrm{i}}\right.$ and $\left.\left[\mathrm{Na}^{+}\right]_{\mathrm{i}}\right)$}

$\left[\mathrm{Ca}^{2+}\right]_{i}$ and $\left[\mathrm{Na}^{+}\right]_{i}$ measurements were conducted on isolated bladder ICC-LCs that were cultured for 2-3 days. Bladder ICC-LCs were washed with Hank's solution (Boster) for $5 \mathrm{~min}$ and then incubated with Fluo-4 AM (for $\left[\mathrm{Ca}^{2+}\right]_{\mathrm{i}}$ measurement, $10 \mu \mathrm{M}$, Molecular Probes, Eugene, OR, USA) or SBFI-AM (for $\left[\mathrm{Na}^{+}\right]_{\mathrm{i}}$ measurement, $10 \mu \mathrm{M}$, Sigma) for $30 \mathrm{~min}$ at $37^{\circ} \mathrm{C}$. After incubation, the bladder ICC-LCs were washed thrice in Hank's solution for $5 \mathrm{~min}$. Bladder ICC-LCs were distinguished according to their distinctive morphology: stellate- or elongatedshaped, with several branches ${ }^{9} . \mathrm{Ca}^{2+}$ and $\mathrm{Na}^{+}$imaging was performed on the laser-scanning confocal microscope (Leica, Germany). The excitation wavelength for $\left[\mathrm{Ca}^{2+}\right]_{\mathrm{i}}$ measurement and $\left[\mathrm{Na}^{+}\right]_{\mathrm{i}}$ measurement was 488 $\mathrm{nm}$ and $405 \mathrm{~nm}$, respectively. After imaging for $1 \mathrm{~min}$, the effects of hypotonic stress with or without GsMTx4 $(2.5 \mu \mathrm{M})$ on bladder ICC-LC real-time $\left[\mathrm{Ca}^{2+}\right]_{\mathrm{i}}$ and $\left[\mathrm{Na}^{+}\right]_{\mathrm{i}}$ were detected, and the effects of hypotonic stress on real-time $\left[\mathrm{Ca}^{2+}\right]_{\mathrm{i}}$ and $\left[\mathrm{Na}^{+}\right]_{\mathrm{i}}$ in small interfering RNA (siRNA)-treated bladder ICC-LCs were also tested. The results are presented as relative fluorescence intensities $(\mathrm{RFI}=\mathrm{F} 1 / \mathrm{F} 0$, where $\mathrm{F} 0$ is the baseline fluorescence intensity before hypotonic stress application, and F1 is the real-time fluorescence intensity after hypotonic stress application).

In the $\left[\mathrm{Ca}^{2+}\right]_{\mathrm{i}}$ and $\left[\mathrm{Na}^{+}\right]_{\mathrm{i}}$ measurements, to activate the Piezo1 channel, we applied hypotonic stress to bladder ICC-LCs similar to previous reports ${ }^{32}$. The Hank's solution used comprised the following (in $\mathrm{mM}$ ): $138 \mathrm{NaCl}$, $5.33 \mathrm{KCl}, 4.17 \mathrm{NaHCO}_{3}, 0.441 \mathrm{KH}_{2} \mathrm{PO}_{4}, 0.338 \mathrm{NaH}_{2} \mathrm{PO}_{4}$, $1.26 \mathrm{CaCl}_{2}, 0.407 \mathrm{MgSO}_{4}, 0.493 \mathrm{MgCl}_{2}$ and $5.56 \mathrm{D}$-glucose, $\mathrm{pH} 7.4(308 \mathrm{mOsm} / \mathrm{L})$. First, we prepared a low $\mathrm{NaCl}$ solution $(113 \mathrm{mOsm} / \mathrm{L})$ by decreasing the $\mathrm{NaCl}$ concentration to $45 \mathrm{mM}$ in the Hank's solution. Isotonic (300 $\mathrm{mOsm} / \mathrm{L})$ and hypotonic $(120 \mathrm{mOsm} / \mathrm{L})$ solutions were prepared by adding $187 \mathrm{mM}$ mannitol and $7 \mathrm{mM}$ mannitol, respectively, to the low $\mathrm{NaCl}$ solution. In the $\left[\mathrm{Ca}^{2+}\right]_{\mathrm{i}}$ and $\left[\mathrm{Na}^{+}\right]_{\mathrm{i}}$ measurements, bladder ICC-LCs were initially maintained in $100 \mu \mathrm{L}$ of isotonic solution $(300 \mathrm{mOsm} / \mathrm{L})$. After imaging for $1 \mathrm{~min}, 100 \mu \mathrm{L}$ of hypotonic solution $(120 \mathrm{mOsm} / \mathrm{L})$ was added to the isotonic solution to obtain the final hypotonic solution $(210 \mathrm{mOsm} / \mathrm{L})$.

\section{Patch-clamp experiments}

Bladder ICC-LCs were routinely cultured for $2-3$ days and identified according to their distinctive morphology, as described above. $\mathrm{I}_{\mathrm{NCX}}$ was recorded in the whole-cell patchclamp mode. Glass electrodes possessing approximate resistances of 4-6 $\mathrm{M} \Omega$ were pulled from a Model P-97 Flaming/Brown Micropipette Puller (Sutter Instrument Co., Novato, CA, USA). For recording, the glass electrodes were filled with pipette solution containing the following compounds (in $\mathrm{mM}$ ): $51 \mathrm{NaCl}, 100 \mathrm{CsOH}, 5 \mathrm{KCl}, 2 \mathrm{MgCl}, 4.94$ $\mathrm{CaCl}_{2}, 20$ tetraethylammonium $\mathrm{Cl}, 10$ 4-(2-hydroxyethyl)-1piperazineethanesulfonic acid (HEPES), 1 adenosine $5^{\prime}$-triphosphate disodium salt hydrate $\left(\mathrm{Na}_{2} \mathrm{ATP}\right), 5$ ethylene glycol tetraacetic acid (EGTA), and 8 D-glucose (pH 7.2). Bladder ICC-LCs were maintained in bath solution containing the following compounds (in $\mathrm{mM}$ ): $137 \mathrm{NaCl}, 5 \mathrm{KCl}$, $1.5 \mathrm{CaCl}_{2}, 1 \mathrm{MgCl}_{2}, 10 \mathrm{HEPES}$, and 10 D-glucose (312 $\mathrm{mOsm} / \mathrm{L}, \mathrm{pH}$ 7.4). To continuously activate the Piezo1 channel with hypotonic stress, the osmotic pressure in the bath solution was changed to $200 \mathrm{mOsm} / \mathrm{L}$ by adjusting the $\mathrm{NaCl}$ concentration to $81 \mathrm{mM}$. GsMTx $4(2.5 \mu \mathrm{M})$ was added to the bath solution to block the Piezol channel. $\mathrm{I}_{\mathrm{NCX}}$ was elicited by a step potential ranging from -100 to $+60 \mathrm{mV}$ in increments of $10 \mathrm{mV}$ for $200 \mathrm{~ms}$, with a holding potential of $-40 \mathrm{mV}$. $\mathrm{I}_{\mathrm{NCX}}$ was amplified and recorded using a HEKA EPC10USB amplifier (HEKA Elektronik, Heidelberg, Germany) and filtered at a threshold frequency of 2.9 $\mathrm{kHz}$. The data were analyzed using FitMaster software (HEKA Elektronik) and the density of $\mathrm{I}_{\mathrm{NCX}}$ was normalized to cell capacitance.

\section{Single-cell RT-PCR}

We performed single-cell RT-PCR to confirm the authenticity of isolated bladder ICC-LCs, according to our 
previous description ${ }^{12}$. After electrophysiological testing, a single bladder ICC-LC was sucked into the recording pipette by applying negative pressure. Subsequently, the collected bladder ICC-LC was expelled instantly into NP40 lysis buffer (Beyotime) containing RNAase inhibitor (Beyotime). After being centrifuged at $4{ }^{\circ} \mathrm{C}$ for $30 \mathrm{~s}$ with 1000 r.p.m., each cell was lysed on ice for $20 \mathrm{~min}$. Singlestranded cDNA was synthesized using a Sensiscript RT Kit (Qiagen, Hilden, Germany) according to the manufacturer's instructions. The primers used in this experiment are listed in Table S1. The thermocycling procedure was set up as follows: $94^{\circ} \mathrm{C}$ for $1 \mathrm{~min}, 94^{\circ} \mathrm{C}$ for $30 \mathrm{~s}, 60^{\circ} \mathrm{C}$ for $30 \mathrm{~s}$, and $72{ }^{\circ} \mathrm{C}$ for $1 \mathrm{~min}$ (40 cycles). The negative control reaction was performed by removing templates. Amplification products were viewed via GoldView (SBS Genetech Co., Beijing, China) staining in 1.5\% agarose gels. Only the $\mathrm{I}_{\mathrm{NCX}}$ values recorded in c-kit-positive cells were included in the statistical analysis.

\section{Co-immunoprecipitation (co-IP) analysis}

Whole bladders removed from rats in the CYP-8d group were separately lysed with RIPA lysis buffer (Beyotime) and centrifuged at $12,000 \mathrm{rpm}$ at $4{ }^{\circ} \mathrm{C}$ for $30 \mathrm{~min}$ to collect the supernatant. The lysates were precleared with Dynabeads Protein G magnetic beads (Thermo Fisher Scientific) at $4{ }^{\circ} \mathrm{C}$ for $15 \mathrm{~min}$ and then incubated with antiPiezo1 (Alomone labs) and anti-NCX1 (Abcam) primary antibodies or negative control IgG overnight at $4{ }^{\circ} \mathrm{C}$. Sequentially, immunocomplexes were precipitated with Dynabeads Protein G magnetic beads. After the beads were washed with PBS containing $0.1 \%(\mathrm{v} / \mathrm{v})$ TX-100 (Beyotime) three times, the precipitates were loaded onto SDS-PAGE gels for western blot analysis.

\section{RNA interference}

To knockdown endogenous Piezo1 channel expression, siRNA targeting rat Piezo1 or negative control siRNA (both purchased from Genema, Shanghai, China) were used to transfect isolated bladder ICC-LCs at $0.2 \mu \mathrm{M}$ concentration with Lipofectamine 2000 (Thermo Fisher Scientific, Waltham, MA, USA). The siRNA sequences were as follows: siRNA-Piezo1, (forward) 5'-CGGCCAACAUAAAGAACA UTT-3' and (reverse) 5'-AUGUUCUUUAUGUUGGCC GTT-3'; negative control siRNA, (forward) 5'-UUCUCCG AACGUGUCACGUTT- $3^{\prime}$ and (reverse) $5^{\prime}$-ACGUGACAC GUUCGGAGAATT-3'. After transfection for $48 \mathrm{~h}$, quantitative RT-PCR and IF staining were used to detect the interference efficiency. siRNA-treated bladder ICC-LCs were subsequently utilized for $\left[\mathrm{Ca}^{2+}\right]_{\mathrm{i}}$ or $\left[\mathrm{Na}^{+}\right]_{\mathrm{i}}$ measurement and electrophysiological recordings.

\section{Statistical analysis}

The data are presented as the mean \pm S.D. Statistical analyses were performed with SPSS 16.0 software (SPSS
Inc., Chicago, IL), and significant differences between each group were calculated using unpaired two-tailed Student's $t$-tests or one-way analysis of variance. $P<0.05$ was considered statistically significant. All experiments were performed with at least three independent replications.

\section{Results \\ Rat models with CYP-induced cystitis were successfully established}

To test whether the CYP-induced cystitis models were successfully established in rats, H\&E staining was primarily performed on rat bladder sections from four groups: control, CYP-4h, CYP-48h, and CYP-8d groups. Compared with that from the control rats, bladder sections from CYP4h and CYP-48h rats (acute cystitis models) exhibited severe edema and hyperemia in the LP, and bladder sections from CYP-8d rats (chronic cystitis model) showed moderate edema and hyperemia in the LP accompanied by urothelial hyperplasia (Fig. 1a). Furthermore, we found that the protein levels of two inflammation-associated cytokines (interleukin-6 (IL-6) and tumor necrosis factor- $\alpha$ (TNF- $\alpha$ )) in CYP-4h, CYP-48h, and CYP-8d rats were all significantly higher than those in the control group, and the increase in CYP-4h and CYP-48h rats was more obvious than that in CYP-8d rats (Fig. 1b, c). Meanwhile, we found that CYP-8d rats exhibited obvious urodynamic abnormalities characterized by a significant increase in MBP and a significant decrease in ICI compared with naive rats (Figure S1 a-c). Furthermore, isolated detrusor strips from CYP-8d rats showed more vigorous spontaneous contractions than those from naive rats (Figure S1d and c). These data indicated that rat models with CYP-induced cystitis were successfully established.

\section{Piezo1 channel expression was significantly increased in bladder ICC-LCs from CYP-48h and CYP-8d rats}

Herein, we first found that protein and mRNA levels of Piezo1 channel were significantly increased in whole bladders from the CYP-48h and CYP-8d rats, and those levels in CYP-8d rat bladders were more prominent. These phenomena were not detected in the CYP-4h rats (Fig. 2a-c). Moreover, consistent results were detected with IHC staining, in which Piezo1 channel expression in bladder sections from CYP-48h and CYP-8d rats was significantly higher than that in bladder sections from control and CYP$4 \mathrm{~h}$ rats, and the changes in CYP-8d rats were more remarkable. Interestingly, we found that increases in Piezo1 channel expression were mainly present in the urothelium and mesenchymal layers (LP and intermuscular region) but not in detrusor smooth muscle cells (Fig. 2d).

To the best of our knowledge, bladder ICC-LCs are located in the LP and intermuscular region, and thus, we further focused on whether Piezo1 channel expression was upregulated in bladder ICC-LCs. The IF staining 

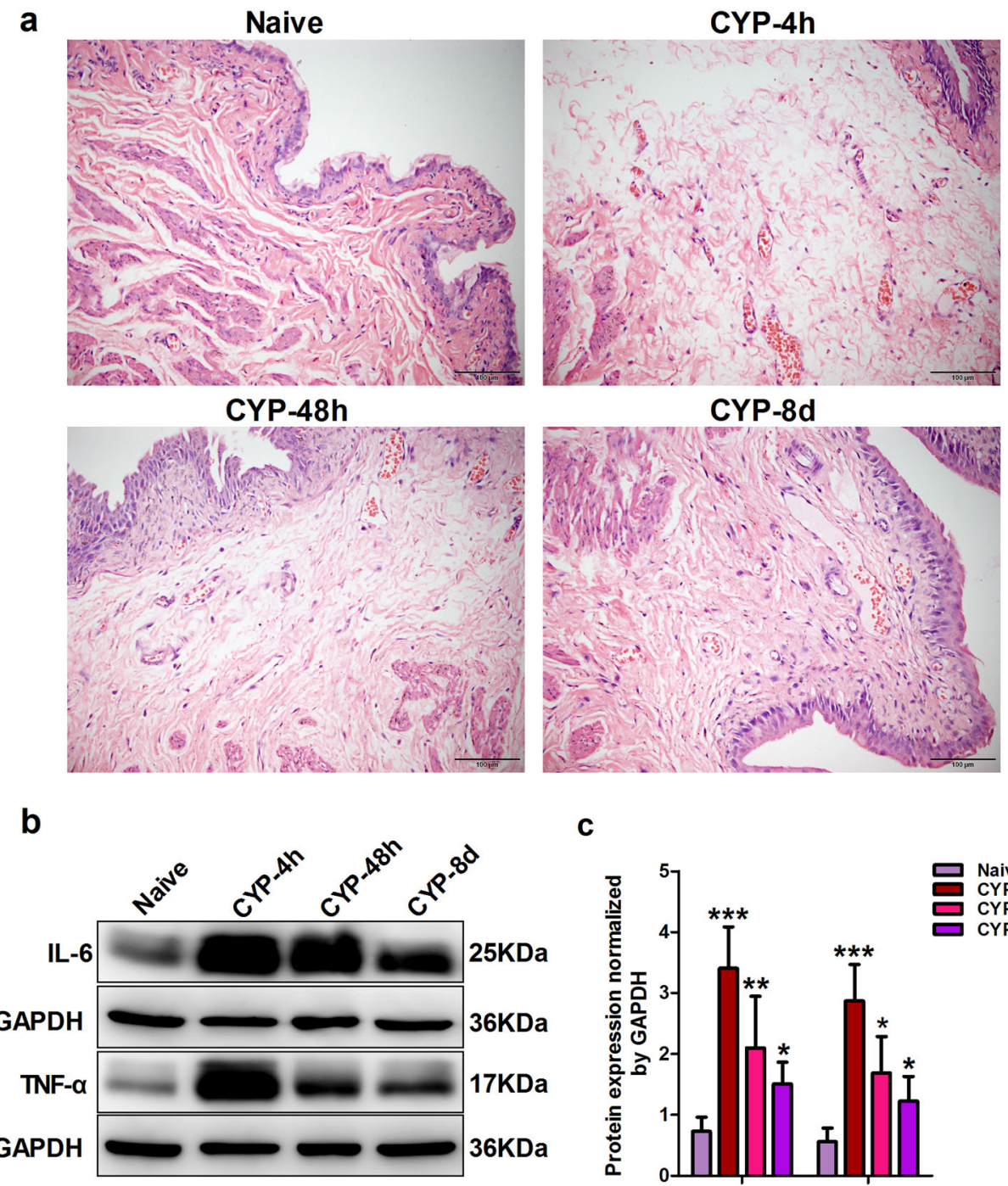

C

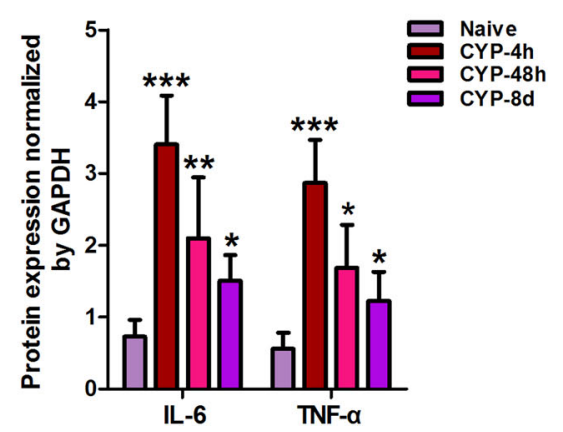

Fig. 1 Cyclophosphamide (CYP) successfully induced acute and chronic cystitis in rats. a Compared with vehicle-treated (naive) rats, characteristic inflammation was detected in histological bladder sections from CYP-treated rats. Bladder sections from CYP-4h and CYP-48h rats (acute cystitis models) demonstrated severe edema and hyperemia in the lamina propria, and bladder sections from CYP-8d rats (chronic cystitis model) exhibited moderate edema and hyperemia in the lamina propria accompanied by urothelial hyperplasia. $\mathbf{b}, \mathbf{c} \| \mathrm{L}-6$ and TNF-a protein expression levels in the bladders from CYP-treated rats were significantly elevated (the data represent the mean \pm S.D., $n=3$; ${ }^{*} P<0.05,{ }^{* *} P<0.01$, and ${ }^{* *} P<0.001$, versus naive rats)

results showed that the Piezo1 channel was expressed in bladder ICC-LCs, and Piezo1 channel expression was significantly enhanced in both the LP ICC-LCs and detrusor ICC-LCs in CYP-48h and CYP-8d rat bladders compared with that in control and CYP-4h rat bladders. Consistently, the increases in bladder ICC-LCs in CYP-8d rats were more prominent (Fig. 2e).

\section{GsMTx4 significantly decreased bladder hyperactivity in CYP-8d rats by inhibiting the Piezo1 channel in bladder ICC-LCs}

We used the Piezo1 channel inhibitor GsMTx4 to test whether increased Piezo1 channel activity was involved in the bladder hyperactivity in CYP-8d rats. Upon GsMTx4 $(2.5 \mu \mathrm{M})$ administration, the MBP was significantly reduced and the ICI was significantly extended in naive and CYP-8d rats. The effects of GsMTx4 administration on MBP and ICI in naive rats were less obvious than in CYP-8d rats (Fig. 3a-c). In contractility studies, we found that GsMTx4 significantly decreased the phasic amplitude of spontaneous contractions in isolated strips from naive and CYP-8d rats. Similarly, the isolated strips from naive rats exhibited lower sensitivity to GsMTx4 than those from CYP-8d rats (Fig. 3d-f). The vehicle (DMSO) had no significant influence on either the urodynamic parameters or the spontaneous contractions in naive and CYP-8d 
a

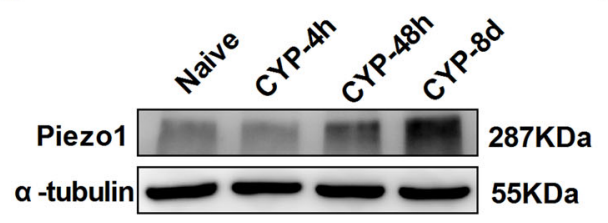

b

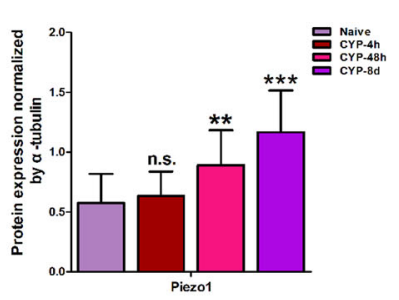

C

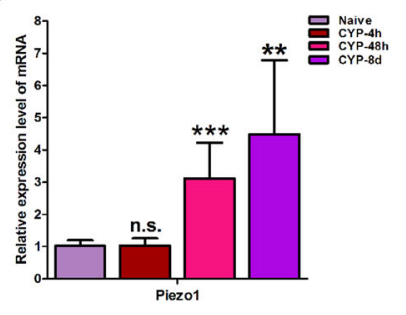

d

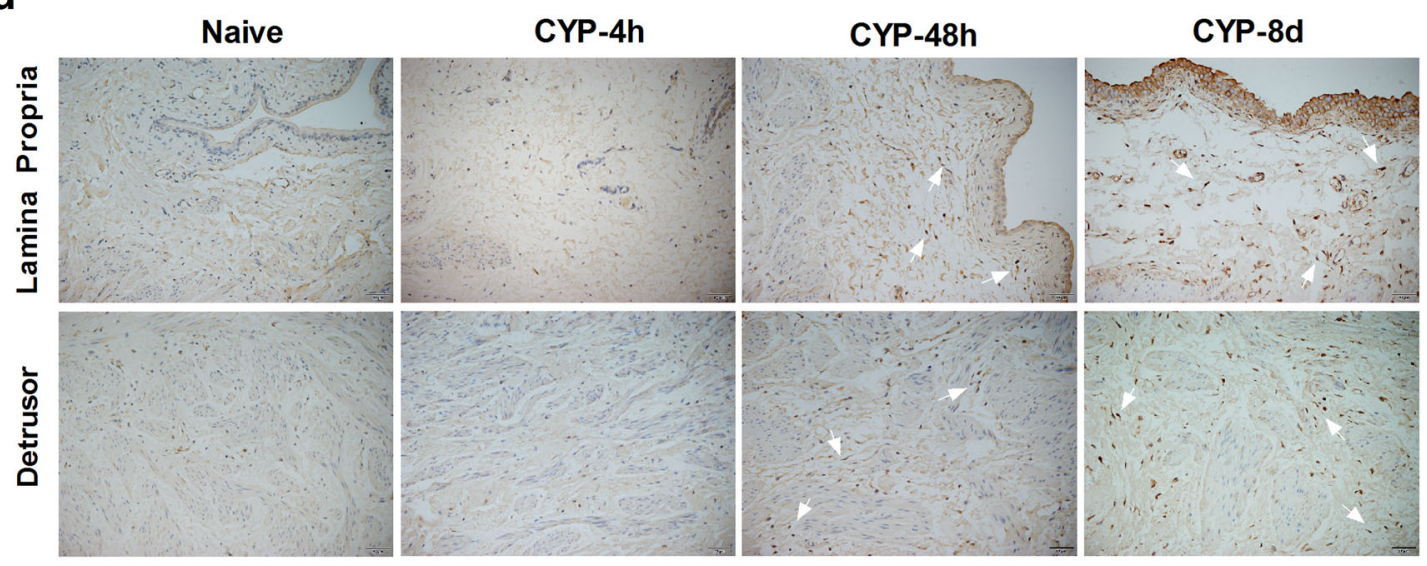

e

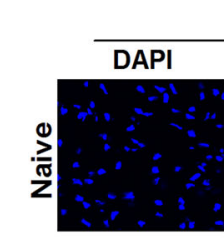

Lamina Propria
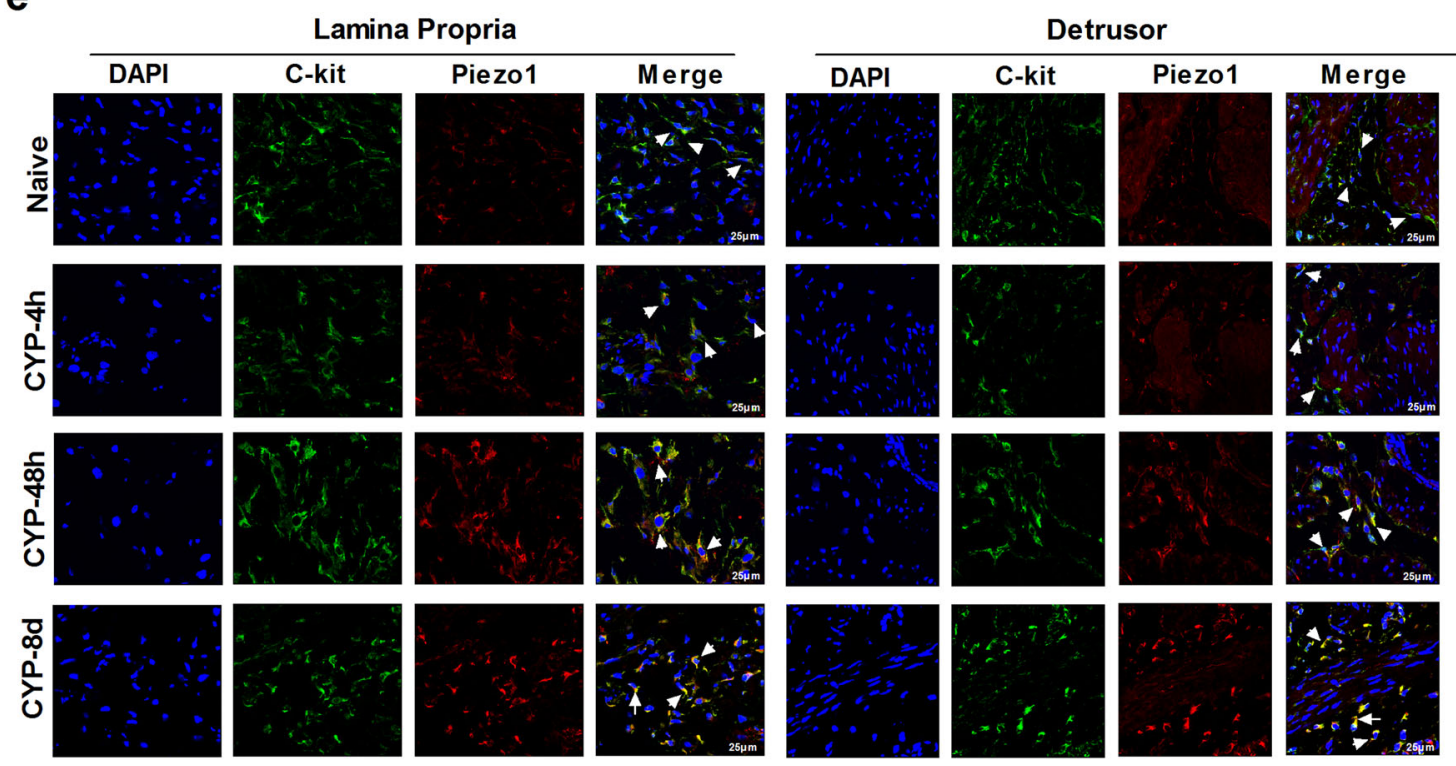

Fig. 2 Piezo1 channel expression was significantly upregulated in CYP-48h and CYP-8d rat bladders. a-c The Piezo1 channel protein and mRNA expression levels were not changed by CYP treatment for $4 \mathrm{~h}$ but were significantly upregulated in whole bladders from CYP-48h and CYP-8d rats, and increases in CYP-8d rat bladders were more remarkable (the data represent the mean \pm S.D., $n=3$; ${ }^{* *} P<0.01$, ${ }^{* * *} P<0.001$, and n.S., no significance, versus naive rats). $\mathbf{d}$ The IHC staining results showed that Piezo 1 channel expression in bladder sections from CYP-48h and CYP-8d rats was significantly increased compared with that in bladder sections from control and CYP-4h rats, and the alterations in CYP-8d rats were more prominent. The increases in Piezo1 channel expression were principally present in the urothelium layer, as well as in the cells located in the lamina propria and intermuscular region (white arrows), but not in detrusor smooth muscle cells. e Double immunostaining of rat bladder sections showed that the Piezo1 channel (red) was expressed in C-kit (green)-positive ICC-LCs (white arrows). Compared with that in control and CYP-4h rat bladders, Piezo1 channel expression was significantly enhanced in both the lamina propria ICC-LCs and detrusor ICC-LCs in CYP-48h and CYP-8d rat bladders. Similarly, bladder ICC-LCs in CYP-8d rats possessed more remarkable increases in Piezo1 channel expression 


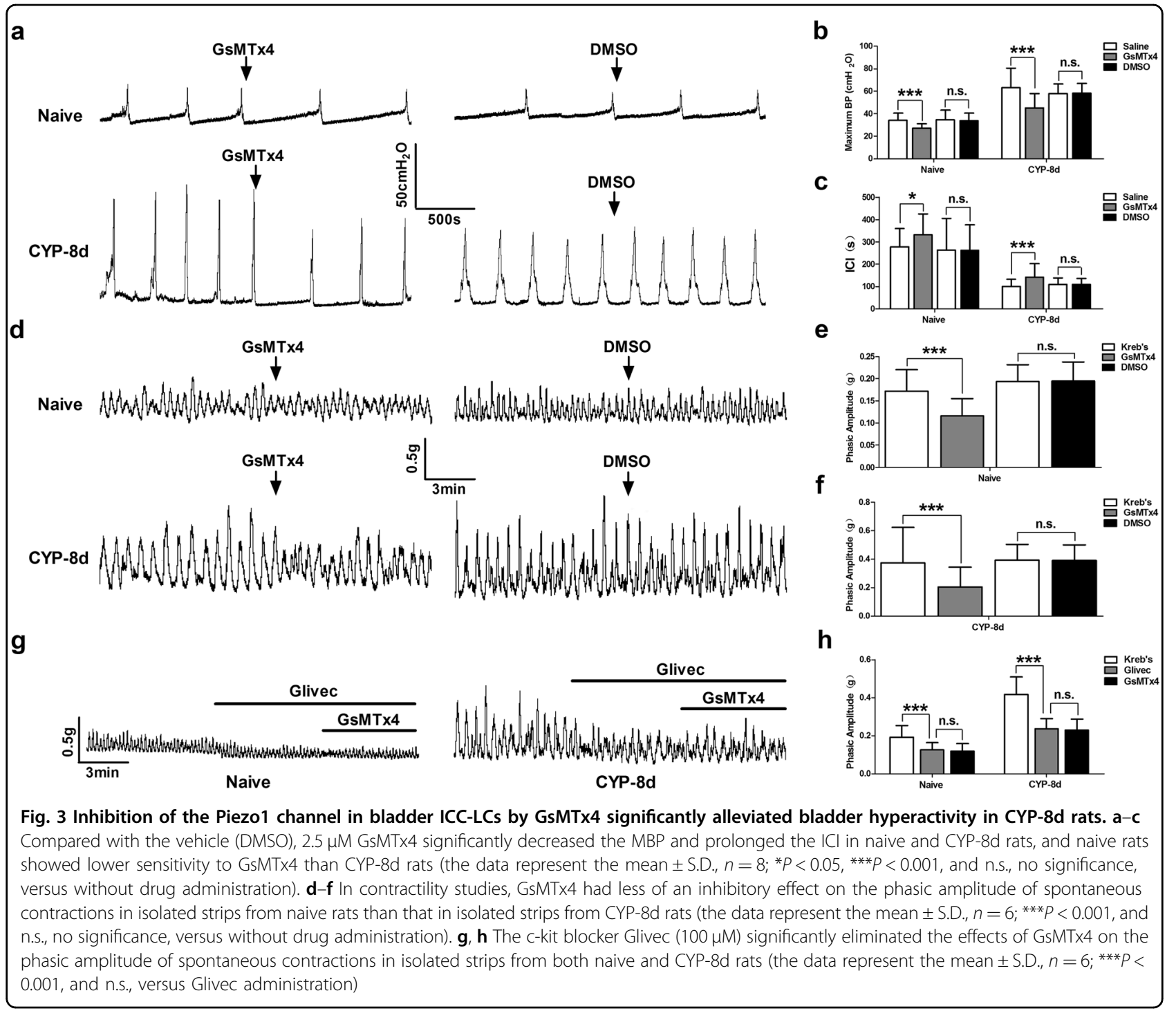

rats. Based on these results, we speculated that the Piezo1 channel plays a more important role in regulating bladder activity in CYP-induced chronic cystitis than under physiologic conditions.

Because the Piezo1 channel was significantly increased in bladder ICC-LCs during cystitis but not in detrusor smooth muscles, we attempted to further identify whether the effects of GsMTx4 on bladder activity were mediated through Piezo1 channel inhibition in bladder ICC-LCs. We utilized the c-kit blocker Glivec to inhibit bladder ICC-LC function as previously reported ${ }^{4}$. We found that upon the application of Glivec $(100 \mu \mathrm{M})$, the effects of GsMTx4 on the phasic amplitude of spontaneous contractions in isolated strips from naive and CYP-8d rats were eliminated (Fig. 3g, h). Based on this result, we suggest that the Piezo1 channel may control bladder activity by regulating bladder ICCLC function.

Piezo1 channel activation significantly enhanced the $\left[\mathrm{Ca}^{2+}\right]_{i}$ and $\left[\mathrm{Na}^{+}\right]_{i}$ in bladder ICC-LCs during CYP-induced chronic cystitis

To further explore the mechanisms underlying the functional role of the Piezo1 channel in regulating bladder ICC-LC function, we focused on the two most important ions associated with cell excitation, $\mathrm{Ca}^{2+}$ and $\mathrm{Na}^{+}$. Hypotonic stress was applied to bladder ICC-LCs to activate the Piezo1 channel. In bladder ICC-LCs from naive and CYP-8d rats, hypotonic stress significantly increased $\left[\mathrm{Ca}^{2+}\right]_{\mathrm{i}}$ and $\left[\mathrm{Na}^{+}\right]_{\mathrm{i}}$, and such increases in both the $\left[\mathrm{Ca}^{2+}\right]_{\mathrm{i}}$ and $\left[\mathrm{Na}^{+}\right]_{\mathrm{i}}$ were significantly weakened by GsMTx4 $(2.5 \mu \mathrm{M})$ (Fig. 4a-d). We speculated that the 


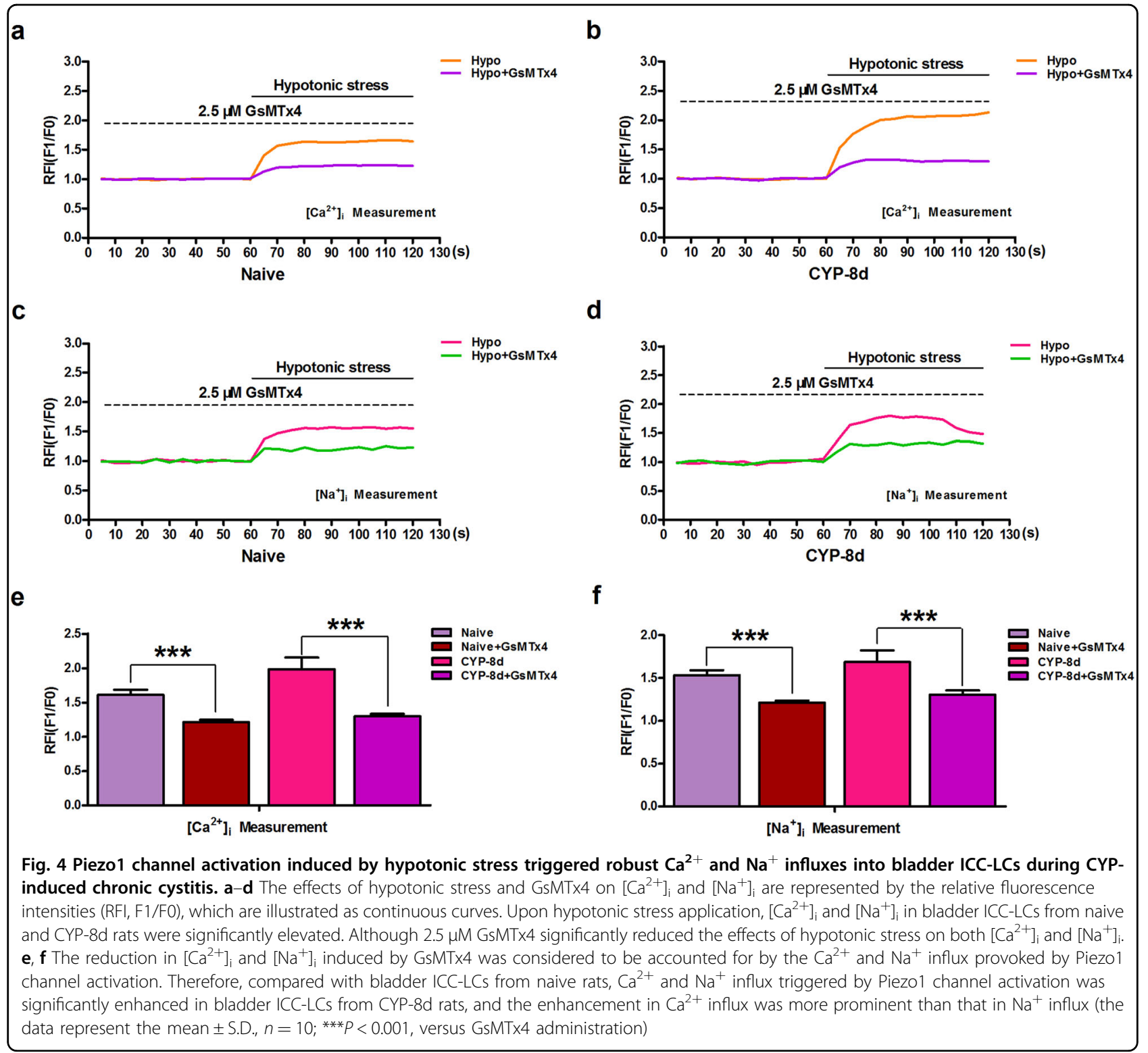

reduction in $\left[\mathrm{Ca}^{2+}\right]_{\mathrm{i}}$ and $\left[\mathrm{Na}^{+}\right]_{\mathrm{i}}$ induced by GsMTx4 accounted for the $\mathrm{Ca}^{2+}$ and $\mathrm{Na}^{+}$influx elicited by Piezo1 channel activation. Therefore, we concluded that the $\mathrm{Ca}^{2+}$ and $\mathrm{Na}^{+}$influx induced by Piezol channel activation was significantly enhanced in bladder ICC-LCs from CYP-8d rats compared with that in bladder ICC-LCs from naive rats, and the enhancement in $\mathrm{Ca}^{2+}$ influx was more remarkable (Fig. 4e, f). Moreover, upon hypotonic stress application, $\left[\mathrm{Ca}^{2+}\right]_{\mathrm{i}}$ continuously rose to a high level in bladder ICC-LCs from CYP-8d rats. However, $\left[\mathrm{Na}^{+}\right]_{\mathrm{i}}$ stably increased followed by a rapid decrease (Fig. 4b, d). A similar phenomenon was not detected in bladder ICCLCs from naive rats. These data prompted us to consider that, in addition to the Piezo1 channel, $\left[\mathrm{Ca}^{2+}\right]_{\mathrm{i}}$ and $\left[\mathrm{Na}^{+}\right]_{\mathrm{i}}$ in bladder ICC-LCs may be regulated by other factors during CYP-induced chronic cystitis.

\section{$\mathrm{Na}^{+} / \mathrm{Ca}^{2+}$ exchanger (NCX) expression was significantly increased and the reverse mode of NCX was relatively activated in bladder ICC-LCs from CYP-8d rats}

NCX is thought to be a key transporter in the regulation of cytosolic $\mathrm{Ca}^{2+}$ dynamics through its ability to transport three $\mathrm{Na}^{+}$ions for every one $\mathrm{Ca}^{2+}$ ion. NCX normally operates to maintain $\mathrm{Ca}^{2+}$ homeostasis by removing $\mathrm{Ca}^{2+}$ from the cells in the forward mode and bringing $\mathrm{Ca}^{2+}$ into the cells in the reverse mode ${ }^{33}$. To determine whether NCX is involved in the control of $\mathrm{Ca}^{2+}$ dynamics in bladder ICC-LCs during CYP-induced chronic cystitis, we 


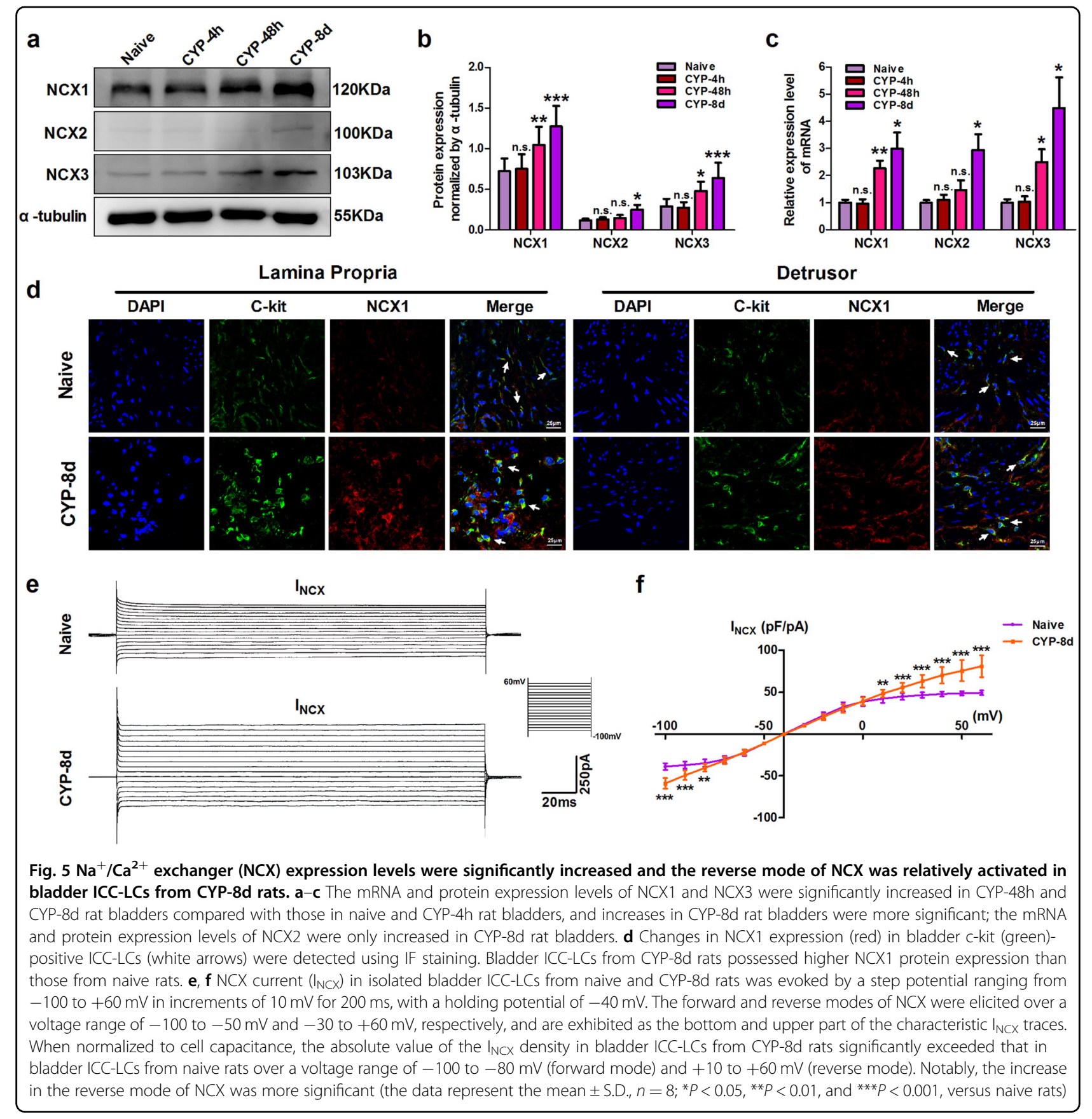

first examined changes in NCX subtype (NCX1-3) expression and function in CYP-8d rats. The results showed that NCX1 and NCX3 mRNA and protein expression was significantly increased in CYP-48h and CYP-8d rat bladders compared with naive and CYP-4h rat bladders, and increases in CYP-8d rat bladders were more significant. NCX2 mRNA and protein expression was only altered in CYP-8d rat bladders (Fig. 5a-c). Based on the fact that NCX1 protein expression was the most dominant in whole rat bladders under both physiologic and pathologic conditions, we proposed that NCX1 may play a vital role in regulating bladder function. Hence, we subsequently identified changes in NCX1 protein expression in bladder ICC-LCs using IF staining. We found that NCX1 protein expression in bladder ICC-LCs from CYP$8 \mathrm{~d}$ rats was significantly higher than that in ICC-LCs from naive rats (Fig. 5d).

Furthermore, we conducted whole-cell patch-clamp experiments to record the $\mathrm{NCX}$ current $\left(\mathrm{I}_{\mathrm{NCX}}\right)$ in isolated bladder ICC-LCs. As shown in Fig. 5e, the $\mathrm{I}_{\mathrm{NCX}}$ was 
recorded in bladder ICC-LCs from naive and CYP-8d rats. When normalized to cell capacitance, the absolute value of $\mathrm{I}_{\mathrm{NCX}}$ density in bladder ICC-LCs from CYP-8d rats was significantly enhanced compared with that in bladder ICC-LCs from naive rats over a voltage range of -100 to $-80 \mathrm{mV}$ (forward mode) and +10 to $+60 \mathrm{mV}$ (reverse mode). In bladder ICC-LCs from CYP-8d rats, the absolute value of $\mathrm{I}_{\mathrm{NCX}}$ density was enhanced (at $-100 \mathrm{mV}$ : naive: $38.82 \pm 4.03$ versus CYP-8d: $59.02 \pm 6.36$; at +60 $\mathrm{mV}$ : naive: $49.07 \pm 2.77$ versus CYP-8d: $80.93 \pm 12.78$ ) (Fig. 5f). We found that the enhancement in the reverse mode of NCX was more significant, which indicated that the reverse mode of NCX was relatively activated in bladder ICC-LCs during CYP-induced chronic cystitis. In addition, the results of single-cell RT-PCR following electrophysiological testing demonstrated that only amplified c-kit (235 bp) and GAPDH (181 bp) products were found in the agarose gel lanes (Figure S2). According to this result, the authenticity of the isolated bladder ICCLCs was confirmed.

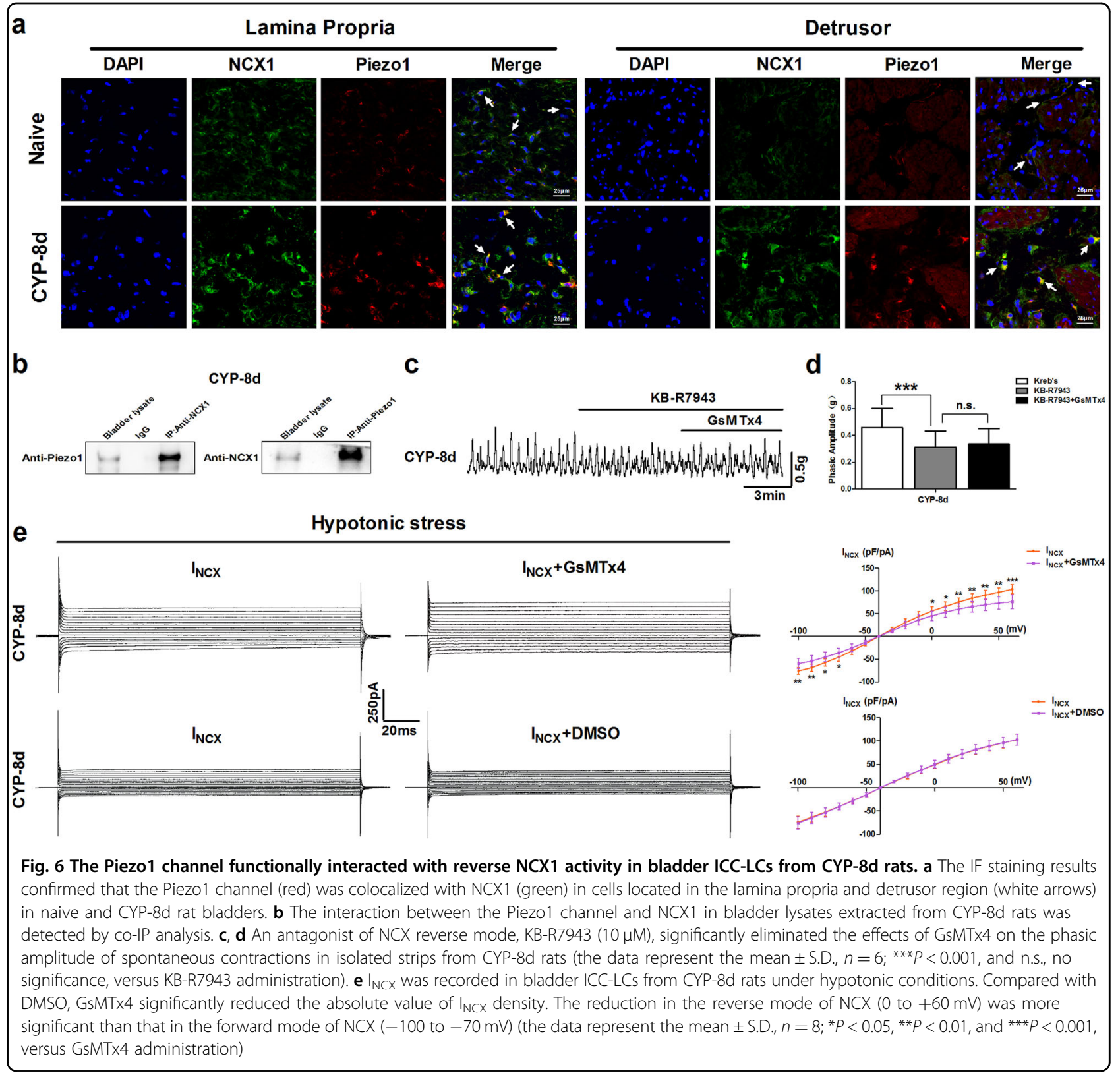




\section{Piezo1 channel functionally interacted with the reverse mode of NCX1 in bladder ICC-LCs from CYP-8d rats}

To further examine the relationships between the Piezo1 channel and NCX1, we performed IF staining and a co-IP analysis. We found that the Piezo1 channel was colocalized with NCX1 in cells located in the LP and detrusor region in naive and CYP-8d rat bladders (Fig. 6a). Moreover, the co-IP analysis results showed that the Piezo1 channel and NCX1 interact with each other in CYP-8d rat bladders (Fig. 6b). Subsequently, we conducted contractility studies and patchclamp experiments to test the functional interaction between the Piezo1 channel and NCX1. After administration of the NCX reverse mode inhibitor KB-R7943 $(10 \mu \mathrm{M})$, GsMTx4 no longer reduced the phasic amplitude of spontaneous contractions in isolated strips from CYP-8d rats (Fig. 6c, d). Furthermore, we found that upon the application of hypotonic stress, the absolute value of the $\mathrm{I}_{\mathrm{NCX}}$ density was significantly increased in bladder ICC-LCs from CYP-8d rats (at $-100 \mathrm{mV}: 75.92 \pm 7.30$; at $+60 \mathrm{mV}: 103.30$ \pm 9.66 versus that in Fig. 5f). GsMTx4 significantly decreased the absolute value of the $\mathrm{I}_{\mathrm{NCX}}$ density (at -100 $\mathrm{mV}: \quad 59.68 \pm 11.06$; at $+60 \mathrm{mV}: 75.87 \pm 15.19$ ), whereas DMSO had no effects on $\mathrm{I}_{\mathrm{NCX}}$ density (Fig. 6e). These results showed that compared with the forward mode of NCX, the reverse mode of NCX was more significantly altered by Piezol channel activation or inhibition in bladder ICC-LCs from CYP-8d rats. Taken together, the results suggest that the Piezo1 channel functionally interacts with the reverse mode of NCX, especially NCX1, in bladder ICCLCs during CYP-induced chronic cystitis.

siRNA targeting the Piezo1 channel significantly reduced $\left[\mathrm{Ca}^{2+}\right]_{\mathrm{i}}$ and $\left[\mathrm{Na}^{+}\right]_{\mathrm{i}}$ and the $\mathrm{I}_{\mathrm{NCX}}$ density in bladder ICC-LCS from CYP-8d rats

To more specifically identify the functional role of the Piezo1 channel in bladder ICC-LCs during CYP-induced chronic cystitis, we utilized siRNA targeting rat Piezo1 (siPiezo1) to knockdown Piezo1 channel expression. Compared with negative control siRNA (ncPiezo1), siPiezo1 significantly decreased Piezo1 channel mRNA and protein expression in bladder ICC-LCs (Fig. 7a, b). After Piezo1 channel knockdown using siPiezol, $\left[\mathrm{Ca}^{2+}\right]_{\mathrm{i}}$ and $\left[\mathrm{Na}^{+}\right]_{i}$ measurements and patch-clamp experiments were performed in bladder ICC-LCs. Similar to GsMTx4, siPiezol attenuated the increases in $\left[\mathrm{Ca}^{2+}\right]_{\mathrm{i}}$ and $\left[\mathrm{Na}^{+}\right]_{\mathrm{i}}$ induced by hypotonic stress application in bladder ICCLCs from naive and CYP-8d rats, and reductions caused by siPiezo1 were more obvious in bladder ICC-LCs from CYP-8d rats (Fig. 7c-f). Hence, we confirmed again that $\mathrm{Ca}^{2+}$ and $\mathrm{Na}^{+}$influx induced by Piezo1 channel activation was significantly stronger in bladder ICC-LCs from CYP$8 \mathrm{~d}$ rats than in bladder ICC-LCs from naive rats. In addition, siPiezo1 significantly reduced the absolute value of the $\mathrm{I}_{\mathrm{NCX}}$ density that was recorded under hypotonic conditions in bladder ICC-LCs from CYP-8d rats (at $-100 \mathrm{mV}$ : ncPiezo1: $75.04 \pm 7.54$ versus siPiezo1: $61.12 \pm$ 8.44; at $+60 \mathrm{mV}$ : ncPiezo1: $103.16 \pm 8.92$ versus siPiezo1: $78.42 \pm 6.88$ ) (Fig. $7 \mathrm{~g}, \mathrm{~h}$ ). We also found that the siPiezo1induced decrease in the reverse mode of NCX was more significant than that in the forward mode of NCX.

Taken together, the results suggested that during CYPinduced chronic cystitis, the significantly upregulated Piezo1 channel in bladder ICC-LCs can be activated by mechanical stimulus (including stretch and hypotonic stress) and subsequently facilitate robust $\mathrm{Ca}^{2+}$ and $\mathrm{Na}^{+}$ influx into bladder ICC-LCs. Meanwhile, the Piezo1 channel functionally interacted with the reverse mode of NCX1 that was relatively activated in bladder ICC-LCs during CYP-induced chronic cystitis. As a consequence, three cytosolic $\mathrm{Na}^{+}$ions that permeated the membrane via the Piezo1 channel were transported out of cells in exchange for one $\mathrm{Ca}^{2+}$ ion brought into the cytoplasm through the reverse mode of NCX1 in bladder ICC-LCs. The synergistic effects of the Piezo1 channel and NCX1 significantly increased $\left[\mathrm{Ca}^{2+}\right]_{\mathrm{i}}$ and led to $\mathrm{Ca}^{2+}$ overload in bladder ICC-LCs, which may consequently induce ICC-LC hyperactivity and chronic cystitisassociated hyperactive bladder (Fig. 8).

\section{Discussion}

In the present study, we demonstrated that increased Piezo1 channel activity in bladder ICC-LCs plays a crucial role in chronic cystitis-associated bladder hyperactivity by regulating bladder ICC-LC function. The potential mechanism in CYP-induced chronic cystitis involves hypotonic stress-induced Piezo1 channel activation that significantly elicits robust $\mathrm{Ca}^{2+}$ and $\mathrm{Na}^{+}$influx into bladder ICC-LCs, and the Piezo1 channel functionally interacts with the relatively activated NCX1 reverse mode, which significantly enhances the intracellular $\mathrm{Ca}^{2+}$ concentration and leads to $\mathrm{Ca}^{2+}$ overload in bladder ICC-LCs.

A previous study reported that Piezol mRNA and protein expression was significantly increased only in the suburothelial and detrusor layers in rats after $\mathrm{PBOO}^{22}$. In the current study, we also demonstrated that Piezo1 protein expression was significantly enhanced in the suburothelial layers (LP) during chronic cystitis. However, in the detrusor region, we found that Piezol protein expression was significantly increased in interstitial cells located in the intermuscular region but not in detrusor smooth muscle cells. Meanwhile, we observed that the Piezo1 channel was significantly overexpressed in the urothelial layer during chronic cystitis. We speculated that such conformity and discrepancy may be ascribed to the complicated regulatory mechanisms of Piezo1 channel expression in diverse pathologic states in various experimental models. Further research is needed to clarify the exact mechanism underlying this phenomenon. 


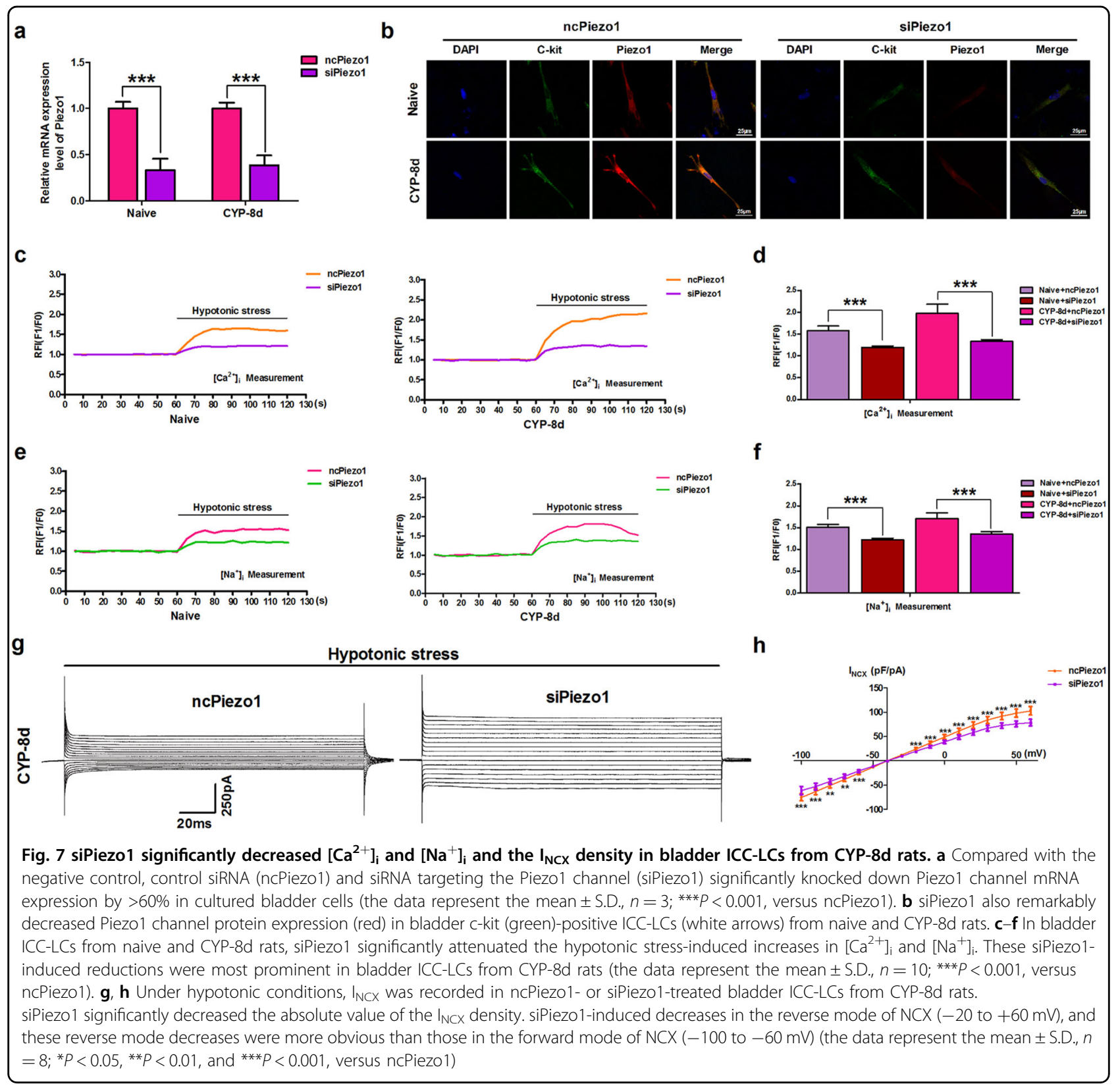

Furthermore, we verified that Piezo1 channel expression was significantly increased in bladder ICC-LCs located in the LP and intermuscular region. This finding prompted us to consider that the Piezo1 channel may participate in chronic cystitis-associated bladder dysfunction by regulating ICC-LC activity. McCloskey and Gurney suggested that bladder ICC-LCs could act as pacemakers to regulate spontaneous detrusor contraction or as intermediaries in the transmission of nerve signals to detrusor smooth muscle cells ${ }^{34}$. Bladder ICC-LCs express the proto-oncogene c-kit, which not only is an identification marker of these cells but also plays a critical role in the control of bladder function ${ }^{35}$. Several studies have reported that selective inhibition of c-kit by imatinib mesylate (Glivec) significantly reduces bladder activity via the c-kit receptor in bladder ICC-LCs in some bladder disorders, including overactive bladder, diabetic cystopathy, and neurogenic bladder ${ }^{5,7}$, 36. c-kit mutation in bladder ICC-LCs is also associated with certain voiding dysfunctions $^{6}$. Based on the functional importance of ICC-LCs with respect to the regulation of bladder function indicated by the above results, although significantly increased Piezo1 channel expression was detected in both the bladder ICC-LCs and the urothelial layer during chronic cystitis, we mainly focused our attention on the functional role of the Piezo1 channel in bladder ICC-LCs. 


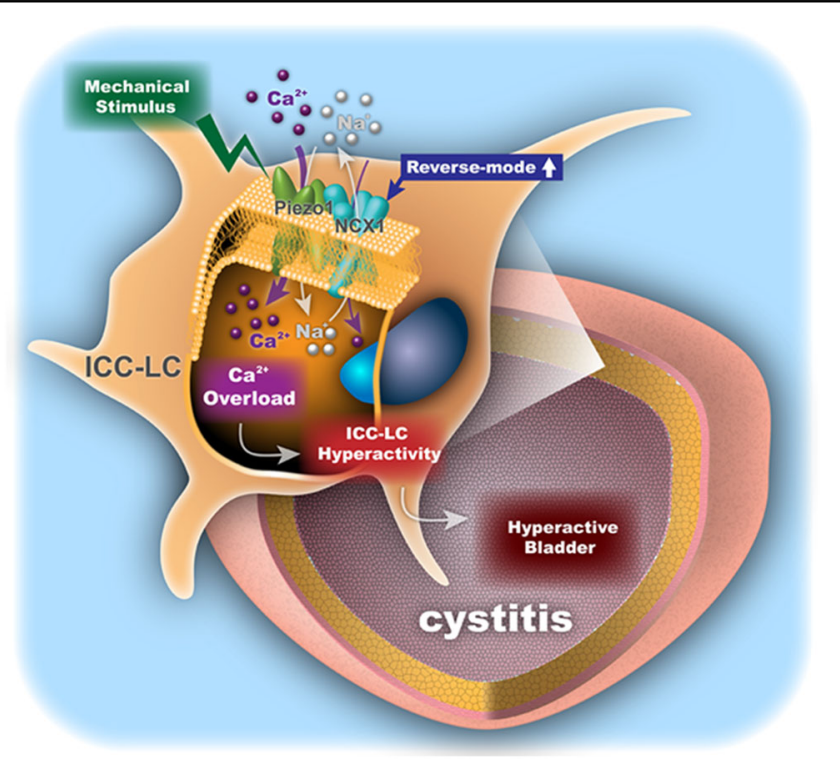

Fig. 8 Schematic representations of the functional roles of the Piezo1 channel and NCX1 in CYP-induced chronic cystitis. In CYP-induced chronic cystitis, when the Piezol channel in bladder ICC-LCs was activated by mechanical stimuli (including stretch and hypotonic stress), robust Ca ${ }^{2+}$ and $\mathrm{Na}^{+}$influx was consequently triggered in bladder ICC-LCs. In addition, the Piezol channel functionally interacted with the relatively activated reverse NCX1 activity in bladder ICC-LCs. As a result, three cytosolic $\mathrm{Na}^{+}$ions permeated through the Piezol channel and were extracted from the bladder ICC-LCs through reverse NCX1 activity in exchange for bringing one $\mathrm{Ca}^{2+}$ ion into the cytoplasm. The synergistic effects of the Piezo1 channel and NCX1 significantly elevated $\left[\mathrm{Ca}^{2+}\right]_{i}$ and led to $\mathrm{Ca}^{2+}$ overload in bladder ICC-LCs, which may consequently result in ICC-LC hyperactivity and chronic cystitis-associated hyperactive bladder

As expected, we subsequently confirmed that the Piezo1 channel blocker GsMTx4 significantly attenuated chronic cystitis-associated bladder hyperactivity by inhibiting the Piezo1 channel in bladder ICC-LCs.

Bladder ICC-LCs possess the ability to generate spontaneous $\mathrm{Ca}^{2+}$ signaling and fire $\mathrm{Ca}^{2+}$ waves in response to cholinergic and purinergic stimuli, which is their functional underpinning for controlling bladder activity 9 . Moreover, it has been shown that $\mathrm{Ca}^{2+}$ signaling in bladder ICC-LCs is upregulated or downregulated in bladder dysfunction associated with suprasacral or sacral spinal cord injury, respectively ${ }^{36}$. In our previous research, we demonstrated that bladder ICC-LC hyperactivity accounted for bladder hyperactivity in CYP-induced chronic cystitis, which was characterized by excessive $\mathrm{Ca}^{2+}$ entry into cells upon carbachol application ${ }^{12}$. Furthermore, $\mathrm{Ca}^{2+}$ overload in cardiomyocytes is closely related to cardiac dysfunction ${ }^{37,38}$. Thus, we suggest that abnormal $\mathrm{Ca}^{2+}$ signaling in bladder ICC-LCs may be involved in the pathogenesis of bladder dysfunction. To the best of our knowledge, the mechanosensitive Piezo1 cation channel may utilize its peripheral domains as force sensors to gate the central ion-conducting pore ${ }^{14}$ and is permeated by a series of ions, including $\mathrm{Ca}^{2+}, \mathrm{Mg}^{2+}, \mathrm{K}^{+}$, and $\mathrm{Na}^{+39}$. In the current study, we found that Piezo1 channel activation induced by hypotonic stress significantly provoked $\mathrm{Ca}^{2+}$ influx in bladder ICC-LCs in
CYP-induced chronic cystitis. Hence, we speculate that the Piezo1 channel is susceptible to being activated during bladder filling or by cellular swelling under cystitis conditions, and Piezo1 channel activation can facilitate robust $\mathrm{Ca}^{2+}$ influx and lead to $\mathrm{Ca}^{2+}$ overload in bladder ICC-LCs during chronic cystitis, which may be an important etiological factor for bladder ICC-LC hyperactivity and the consequent chronic cystitis-associated bladder hyperactivity.

Piezo1 channel activation also significantly provoked $\mathrm{Na}^{+}$influx into bladder ICC-LCs, but the elevated intracellular $\mathrm{Na}^{+}$concentration was subsequently mediated. We speculated that NCX may be involved in this process. NCX plays a central role in cellular $\mathrm{Ca}^{2+}$ homeostasis by removing $\mathrm{Ca}^{2+}$ through its forward mode under physiological conditions ${ }^{33}$. However, augmented $\mathrm{Ca}^{2+}$ entry through reverse NCX activity is responsible for arrhythmia associated with heart failure or ischemia/reperfusion injury ${ }^{40}$. Furthermore, the accelerated $\mathrm{Ca}^{2+}$ influx (reverse) mode of NCX in smooth muscle can lead to an overactive bladder ${ }^{41}$. Consistently, we also observed that the reverse mode of NCX was relatively activated in bladder ICC-LCs during chronic cystitis. Using GsMTx4 or siRNA targeting Piezo1, we confirmed that the Piezo1 channel functionally interacted with the reverse mode of NCX. Through these functional interactions, $\mathrm{Na}^{+}$introduced by the Piezo1 channel will be extracted from the 
cells via reverse NCX activity, especially that of NCX1, which can aggravate $\mathrm{Ca}^{2+}$ overload in bladder ICC-LCs. In addition, the Piezo1 channel was found to be colocalized with NCX1 in cells located in the LP and detrusor region. Based on the finding that the Piezo1 channel and NCX1 were both expressed in bladder ICC-LCs, we speculated that the Piezo1 channel was colocalized with NCX1 in bladder ICC-LCs. Moreover, we found that the Piezo1 channel bound to NCX1. These relationships between the Piezo1 channel and NCX1 can provide the structural foundation for their functional interaction in bladder ICC-LCs.

In the present study, we examined the functional role of the Piezol channel in bladder ICC-LCs in CYP-induced chronic cystitis, and the results will serve as a potent supplement to clarify the comprehensive mechanisms underlying the control of bladder activity by the Piezo1 channel under pathologic conditions. However, a limitation of our study is that we did not test the functional effects of the Piezo2 channel on bladder function regulation. Additional research is required to elucidate the functional role of the Piezo2 channel in regulating bladder activity. Furthermore, it is commonly known that the sarcoplasmic reticulum (SR) plays an important role in regulation of cytosolic $\mathrm{Ca}^{2+}$ dynamics ${ }^{42}$, and further studies are needed to explore the functional relationship between the Piezo1 channel and SR in CYP-induced chronic cystitis or other bladder disorders.

In summary, our study reveals an important interplay among Piezo1 and the NCX1 channel in CYP-induced chronic cystitis. The synergistic effects of the Piezo1 channel and NCX1 significantly increased the intracellular $\mathrm{Ca}^{2+}$ concentration and led to $\mathrm{Ca}^{2+}$ overload in bladder ICC-LCs, which are essential for chronic cystitisassociated bladder hyperactivity. These findings indicate the functional importance of the Piezol channel and NCX1 in bladder ICC-LCs during CYP-induced chronic cystitis, as well as their potential as therapeutic targets for chronic cystitis-associated bladder hyperactivity.

\section{Acknowledgements}

This work was supported by the National Natural Science Foundation of China (nos. 81770761 and 81500580).

\section{Author details}

'Department of Urology, Second Affiliated Hospital, Third Military Medical University, Chongqing 400037, China. ${ }^{2}$ Cancer Center, Institute of Cancer Stem Cell, Dalian Medical University, Dalian 116044, China. ${ }^{3}$ Division of Radiation and Cancer Biology, Department of Radiation Oncology, University of Michigan, Ann Arbor, MI 48105, USA

\section{Authors' contributions}

Q.L. performed the major experiments and drafted the manuscript. J.X. and Z.Y. analyzed the data. B.S. and J.Z. were involved in the H\&E staining, western blotting, quantitative PCR, and immunostaining. Q.W. and X.H. participated in the urodynamic measurements, contractility studies, $\left[\mathrm{Ca}^{2+}\right]_{i}$ and $\left[\mathrm{Na}^{+}\right]_{i}$ measurements, and patch-clamp experiments. F.A. drew the schematic representation. L.L. and M.T. designed the study and edited the paper. All authors reviewed and approved the submission of the paper.

\section{Conflict of interest}

The authors declare that they have no conflict of interest.

\section{Publisher's note}

Springer Nature remains neutral with regard to jurisdictional claims in published maps and institutional affiliations.

Supplementary information accompanies this paper at https://doi.org/ 10.1038/s12276-018-0088-z.

Received: 21 November 2017 Revised: 31 January 2018 Accepted: 14 February 2018.

Published online: 7 May 2018

\section{References}

1. de Oliveira, M. G. et al. Activation of soluble guanylyl cyclase by BAY 58-2667 improves bladder function in cyclophosphamide-induced cystitis in mice. Am. J. Physiol. Ren. Physiol. 311, F85-F93 (2016).

2. LV, J. W. et al. Inhibition of microRNA-214 promotes epithelial-mesenchymal transition process and induces interstitial cystitis in postmenopausal women by upregulating Mfn2. Exp. Mol. Med. 49, e357 (2017).

3. Neuhaus, J. \& Schwalenberg, T. Intravesical treatments of bladder pain syndrome/interstitial cystitis. Nat. Rev. Urol. 9, 707-720 (2012).

4. Kubota, Y., Biers, S. M., Kohri, K. \& Brading, A. F. Effects of imatinib mesylate (Glivec) as a c-kit tyrosine kinase inhibitor in the guinea-pig urinary bladder. Neurourol. Urodyn. 25, 205-210 (2006).

5. Biers, S. M., Reynard, J. M., Doore, T. \& Brading, A. F. The functional effects of a c-kit tyrosine inhibitor on guinea-pig and human detrusor. BJU Int. 97, 612-616 (2006).

6. Okada, S. et al. Attenuation of bladder overactivity in KIT mutant rats. BJU Int. 108, E97-E103 (2011).

7. Vahabi, B., McKay, N. G., Lawson, K. \& Sellers, D. J. The role of c-kit-positive interstitial cells in mediating phasic contractions of bladder strips from streptozotocin-induced diabetic rats. BJU Int. 107, 1480-1487 (2011).

8. Juszczak, K, Maciukiewicz, P., Drewa, T. \& Thor, P. J. Cajal-like interstitial cells as a novel target in detrusor overactivity treatment: true or myth? Cent. Eur. J. Urol. 66, 413-417 (2014).

9. McCloskey, K. D. Interstitial cells in the urinary bladder-localization and function. Neurourol. Urodyn. 29, 82-87 (2010).

10. Gevaert, T. et al. Identification of different phenotypes of interstitial cells in the upper and deep lamina propria of the human bladder dome. J. Urol. 192, 1555-1563 (2014).

11. McCloskey, K. D. Bladder interstitial cells: an updated review of current knowledge. Acta Physiol. (Oxf.). 207, 7-15 (2013).

12. Liu, Q. et al. Cyclophosphamide-induced HCN1 channel upregulation in interstitial Cajal-like cells leads to bladder hyperactivity in mice. Exp. Mol. Med. 49, e319 (2017)

13. Coste, B. et al. Piezo1 and Piezo2 are essential components of distinct mechanically activated cation channels. Science 330, 55-60 (2010).

14. $\mathrm{Ge}, \mathrm{J}$. et al. Architecture of the mammalian mechanosensitive Piezo1 channel. Nature 527, 64-69 (2015).

15. Wang, S. et al. Endothelial cation channel PIEZO1 controls blood pressure by mediating flow-induced ATP release. J. Clin. Invest. 126, 4527-4536 (2016).

16. Gudipaty, S. A. et al. Mechanical stretch triggers rapid epithelial cell division through Piezo1. Nature 543, 118-121 (2017).

17. Albuisson, J. et al. Dehydrated hereditary stomatocytosis linked to gain-offunction mutations in mechanically activated PIEZO1 ion channels. Nat. Commun. 4, 1884 (2013).

18. Glogowska, E. et al. Novel mechanisms of PIEZO1 dysfunction in hereditary xerocytosis. Blood 130, 1845-1856 (2017).

19. Fotiou, E. et al. Novel mutations in PIEZO1 cause an autosomal recessive generalized lymphatic dysplasia with non-immune hydrops fetalis. Nat. Commun. 6, 8085 (2015)

20. Liang, J. et al. Stretch-activated channel Piezo1 is up-regulated in failure heart and cardiomyocyte stimulated by Angll. Am. J. Transl. Res. 9, 2945-2955 (2017) 
21. Miyamoto, T. et al. Functional role for Piezo1 in stretch-evoked $\mathrm{Ca}(2)^{(+)}$influx and ATP release in urothelial cell cultures. J. Biol. Chem. 289, 16565-16575 (2014).

22. Michishita, M., Yano, K., Tomita, K. I., Matsuzaki, O. \& Kasahara, K. I. Piezo1 expression increases in rat bladder after partial bladder outlet obstruction. Life. Sci. 166, 1-7 (2016).

23. Lee, G., Romih, R. \& Zupancic, D. Cystitis: from urothelial cell biology to clinical applications. Biomed. Res. Int. 2014, 473536 (2014).

24. Tyagi, P., Hsieh, V. C., Yoshimura, N., Kaufman, J. \& Chancellor, M. B. Instillation of liposomes vs dimethyl sulphoxide or pentosan polysulphate for reducing bladder hyperactivity. BJU Int. 104, 1689-1692 (2009).

25. Charrua, A. et al. Co-administration of transient receptor potential vanilloid 4 (TRPV4) and TRPV1 antagonists potentiate the effect of each drug in a rat model of cystitis. BJU Int. 115, 452-460 (2015).

26. Zhang, L. et al. An immunogenic peptide, T2 induces interstitial cystitis/painful bladder syndrome: an autoimmune mouse model for interstitial cystitis/ painful bladder syndrome. Inflammation 40, 2033-2041 (2017).

27. Coelho, A. et al. Urinary bladder inflammation induces changes in urothelial nerve growth factor and TRPV1 channels. Br. J. Pharmacol. 172, 1691-1699 (2015).

28. Takahara, Y. et al. Expression of pancreatitis associated proteins in urothelium and urinary afferent neurons following cyclophosphamide induced cystitis. J. Urol. 179, 1603-1609 (2008).

29. Zhang, Q. H., Zhou, Z. S., Lu, G. S., Song, B. \& Guo, J. X. Melatonin improves bladder symptoms and may ameliorate bladder damage via increasing $\mathrm{HO}-1$ in rats. Inflammation 36, 651-657 (2013).

30. Fischer, A. H., Jacobson, K. A., Rose, J. \& Zeller, R. Hematoxylin and eosin staining of tissue and cell sections. CSH Protoc. 2008, pdb.prot4986 (2008).

31. $\mathrm{Tu}, \mathrm{H}$. et al. Serotonin $(5-\mathrm{HT}) 2 \mathrm{~A} / 2 \mathrm{C}$ receptor agonist (2,5-dimethoxy-4-idophenyl)-2-aminopropane hydrochloride (DOI) improves voiding efficiency in the diabetic rat. BJU Int. 116, 147-155 (2015).
32. Soya, M. et al. Plasma membrane stretch activates transient receptor potential vanilloid and ankyrin channels in Merkel cells from hamster buccal mucosa. Cell Calcium 55, 208-218 (2014).

33. Ander, B. P. et al. Differential sensitivities of the NCX1.1 and NCX1.3 isoforms of the $\mathrm{Na}^{+}-\mathrm{Ca}_{2}^{+}$exchanger to alpha-linolenic acid. Cardiovasc. Res. 73, 395-403 (2007).

34. McCloskey, K. D. \& Gurney, A. M. Kit positive cells in the guinea pig bladder. J. Urol. 168, 832-836 (2002).

35. Kubota, Y. et al. Role of KIT-positive interstitial cells of Cajal in the urinary bladder and possible therapeutic target for overactive bladder. Adv. Urol 2011, 816342 (2011).

36. Deng, J. et al. The effects of Glivec on the urinary bladder excitation of rats with suprasacral or sacral spinal cord transection. J. Surg. Res. 183, 598-605 (2013).

37. Kojima, A., Kitagawa, H., Omatsu-Kanbe, M., Matsuura, H. \& Nosaka, S Sevoflurane protects ventricular myocytes against oxidative stressinduced cellular $\mathrm{Ca}^{+}{ }^{+}$overload and hypercontracture. Anesthesiology 119, 606-620 (2013).

38. Zhao, Y. et al. Shensong Yangxin capsules prevent ischemic arrhythmias by prolonging action potentials and alleviating $\mathrm{Ca}_{2}^{+}$overload. Mol. Med. Rep. 13 5185-5192 (2016).

39. Gnanasambandam, R., Bae, C., Gottlieb, P. A. \& Sachs, F. lonic selectivity and permeation properties of human PIEZO1 channels. PLOS. ONE. 10, e0125503 (2015).

40. Nagy, N. et al. Selective $\mathrm{Na}(+) / \mathrm{Ca}(2+)$ exchanger inhibition prevents $\mathrm{Ca}(2+)$ overload-induced triggered arrhythmias. Br. J. Pharmacol. 171, 5665-5681 (2014).

41. Yamamura, $\mathrm{H}$. et al. Overactive bladder mediated by accelerated $\mathrm{Ca} 2+$ influx mode of $\mathrm{Na}+/ \mathrm{Ca} 2+$ exchanger in smooth muscle. Am. J. Physiol. Cell. Physiol. 305, C299-C308 (2013).

42. Zhang, $\mathrm{H}$. et al. STIM1-Ca2 + signaling modulates automaticity of the mouse sinoatrial node. Proc. Natl. Acad. Sci. USA 112, E5618-E5627 (2015). 